\title{
The Controllable Synthesis and SERS Studies of Hollow Urchin-like Gold Nanoparticles
}

\author{
Mingyue Zhang ${ }^{a}$, Weiping Qian ${ }^{\mathrm{b},{ }^{*}}$ \\ State Key Laboratory of Bioelectronics, School of Biological Science and Medical Engineering, \\ Southeast University, Nanjing 210096, China \\ amyz_seu@163.com, bwqian@seu.edu.cn \\ ${ }^{*}$ Corresponding author
}

Keywords: Hollow urchin-like gold nanoparticles, SERS, Controllable synthesis.

\begin{abstract}
Surface-enhanced Raman scattering (SERS) is widely used to provide chemical and physical information, causing widespread attention in many fields, such as biochemical and bioscience fields. To get high SERS signals, noble nanoparticles are applied in SERS detection. Hollow urchin-like gold nanoparticles (HU-GNPs) are a novel nanostructure, which contains rough surfaces and sharp branches. Owing to "Hot spots" effect, it can enhance the surrounding electromagnetic field, generating good SERS effects. In this paper, using seed-mediated growth method, hollow urchin-like gold nanoparticles were prepared. Besides, controllable synthesis was achieved to get the optimal HU-GNPs with good morphology, uniformity and SERS enhancement. And then, SERS substrates were built with HU-GNPs by electrostatic self-assembly method. The SERS signals of NBA can be detected at different concentrations from 10-5 M to 10-9 M. At the same time, field emission scanning electron microscopy (SEM), transmission electron microscopy (TEM), and surface enhanced Raman scattering (SERS) were used for the characterization of the nanoparticles.
\end{abstract}

\section{Introduction}

Since 1970, surface enhanced Raman scattering (SERS) has been increasingly used in many analytical fields as a highly sensitive detection technique. It can provide the information of molecular internal structures by its finger-print identification characteristics [1]. Besides, compared with traditional Raman detection, the SERS signals could be amplified by several orders of magnitude due to the local electromagnetic field enhancement of noble metal nanoparticles.

With the continuous exploration and discovery of researchers, nanoparticles [2], especially noble metal nanoparticles, have unique chemical, optical, electrical and thermal properties and could be broadly applied in biochemical areas [3]. Among them, as the reason of the sharp branches and the complex rough surface, three-dimensional nanoparticles possess good SERS effect [4].

It was found that the rough surface of HU-GNPs can cause electromagnetic enhancement due to the "hot spot" effect [5]. In addition, their advantages are as follows: Firstly, the preparation method is relatively simple, and the synthetic nanoparticles possess good morphology and uniformity. Secondly, compared with silver nanoparticles, gold nanoparticles have better stability and bio-compatibility. Thirdly, they can be further applied to subsequent research after surface modification [6-8].

\section{Experimental}

\subsection{Materials}

trisodium citrate $\left(\mathrm{C}_{6} \mathrm{H}_{5} \mathrm{Na}_{3} \mathrm{O}_{7} \cdot 2 \mathrm{H}_{2} \mathrm{O}\right), \mathrm{AgNO}_{3}, \mathrm{HCl}$, dopamine hydrochloride, anhydrous ethanol, chloroauric acid terahydrate $\left(\mathrm{HAuCl}_{4} \cdot 4 \mathrm{H}_{2} \mathrm{O}\right)$, polyvinyl pyrrolidone (PVP), Nile blue A (NBA) were all purchased from Sinopharm Chemical Reagent Co., Ltd. Water used in the experiments was ultrapure water (resistance, $18 \mathrm{M} \Omega \mathrm{cm}^{-1}$ ). 


\subsection{Synthesis of Ag seeds}

$9 \mathrm{mg} \mathrm{AgNO}_{3}$ was dissolved in $50 \mathrm{~mL}$ water, subsequently heating to boil. Then, $1.2 \mathrm{~mL}$ trisodium citrate $\left(1 \%\right.$ in mass) was added for the reduction reaction of $\mathrm{AgNO}_{3}$. After 40 minutes, the collargol was collected and cooled to room temperature for the next step.

\subsection{Controllable synthesis of HU-GNPs}

Add $20 \mathrm{~mL}$ ultrapure water in a $50 \mathrm{~mL}$ beaker, placed on a magnetic stirrer. Then $100 \mu \mathrm{L} \mathrm{Ag} \mathrm{seeds}$ solution was added under $40{ }^{\circ} \mathrm{C}$ water bath. After the solution was intensively mixed, $500 \mu \mathrm{L} \mathrm{HAuCl}_{4}$ solution (1\% in mass) was dropwise added, and $25 \mu \mathrm{L} \mathrm{HCl}$ was added later. After thoroughly stirring for $10 \mathrm{~min}, 5 \mathrm{~mL} 12 \mathrm{mM}$ dopamine hydrochloride solution and $5 \mathrm{~mL} \mathrm{PVP} \mathrm{(1 \%} \mathrm{in} \mathrm{mass)} \mathrm{were} \mathrm{injected.}$ The solution color would turn orange after it reacted for 20 minutes. And the solution was placed under room temperature overnight. Finally, HU-GNPs were collected after being centrifuged and washed three times.

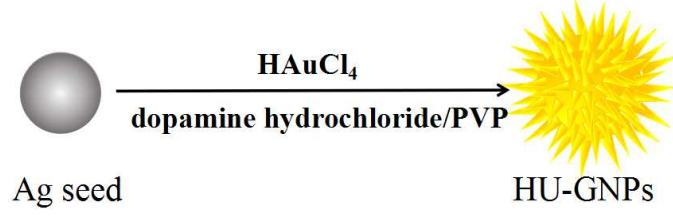

Fig. 1. The schematic of the synthesis of HU-GNPs.

\subsection{Results and discussion}

\subsubsection{The effect of different Ag seeds volume on HU-GNPs}

As shown in Figure 2 (A-D), with the Ag seeds volume in the reaction system changing, the morphology of HU-GNPs were different. In the experiment, after Ag seeds were added, gold nanoparticles with hollow urchin-like structures were produced. Figure 2E shows the different SERS spectra of the above nanoparticles, of which the HU-GNPs synthesized with $100 \mu \mathrm{L} \mathrm{Ag}$ seeds have strong SERS effect.
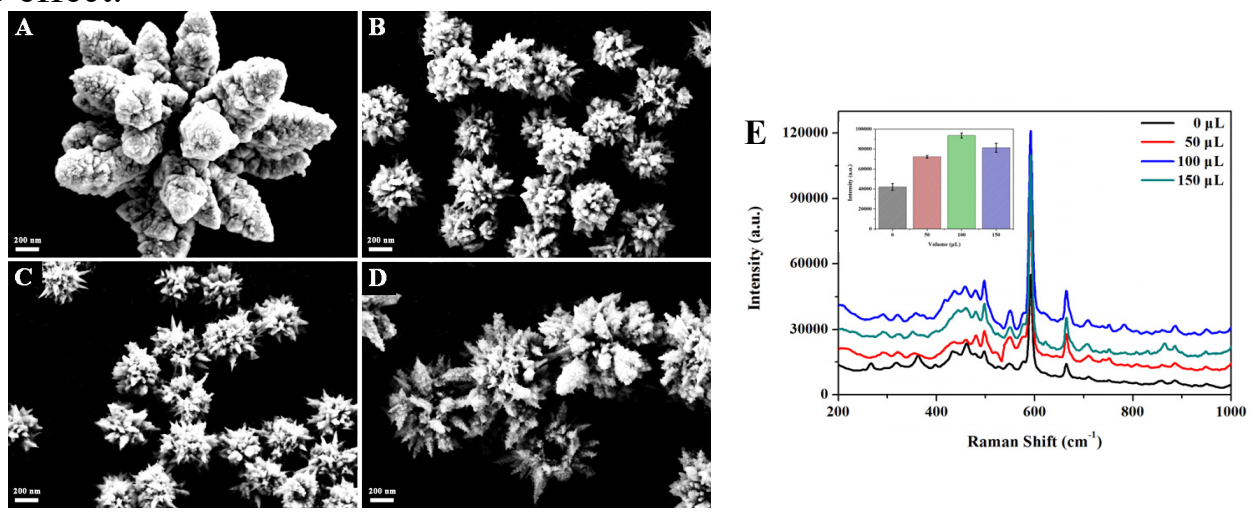

Fig. 2. The SEM images of the representatives of HU-GNPs with different Ag seed volume. (A) $50 \mu \mathrm{L}$, (B) $100 \mu \mathrm{L}$, (C)

$150 \mu \mathrm{L}$, (D) $200 \mu \mathrm{L}$. (E) The SERS spectra of hollow urchin-like gold nanoparticles with different Ag seed volume (10-6 M NBA). The illustration was the SERS signals strength at $592 \mathrm{~cm}-1$.

\subsubsection{The effect of different $\mathrm{HAuCl} 4$ volume on $\mathrm{HU}-\mathrm{GNPS}$}

The morphology of the nanoparticles changes, when adding different $\mathrm{HAuCl}_{4}$ volume (Figure 3). Within a certain range, the branches of HU-GNPs gradually grew with the increasing of $\mathrm{HAuCl}_{4}$ volume. The reason might be that the reducing agents in the reaction system could reduce Au in this range. Thus, it promoted the growth of the branches. When the $\mathrm{HAuCl}_{4}$ is excessive, the reducing agents in the reaction system were not enough to reduce $\mathrm{Au}$, resulting the shorter branches. 

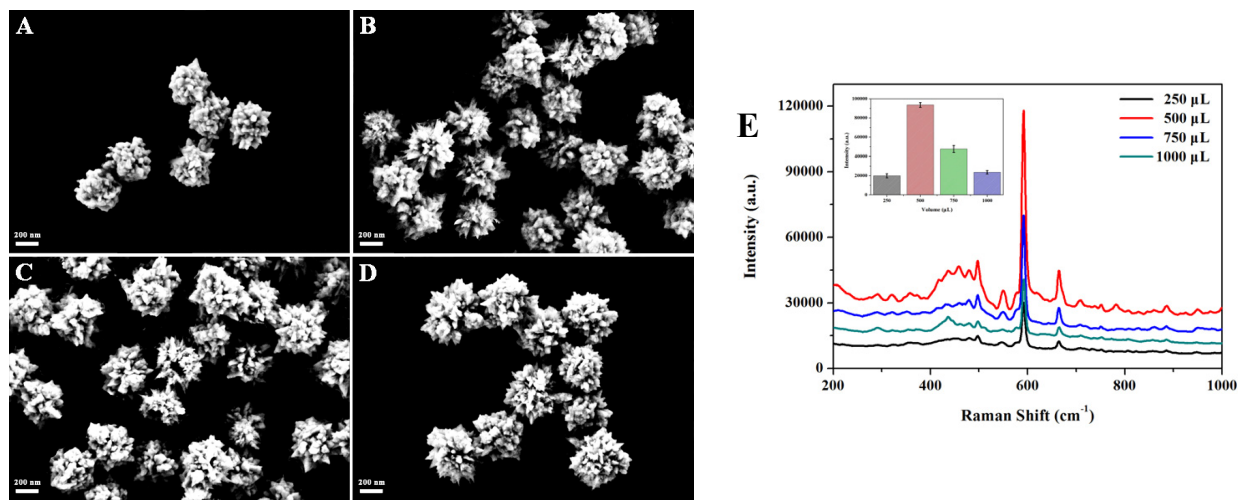

Fig. 3. The SEM images of the representatives of HU-GNPs with different HAuCl4 volume. (A) $250 \mu \mathrm{L}$; (B) $500 \mu \mathrm{L}$;

(C) $750 \mu \mathrm{L}$; (D) $1000 \mu \mathrm{L}$. (E) The SERS spectra of hollow urchin-like gold nanoparticles with different HAuCl4 volume (10-6 M NBA). The illustration was the SERS signals strength at $592 \mathrm{~cm}-1$.

\subsubsection{The effect of different bath temperature on HU-GNPS}

With the increasing of the bath temperature, the branches of HU-GNPs were more obvious and sharp. However, when the temperature was too high, the nanoparticles would be out of shape. The reason might be that the heating accelerated the displacement reaction.
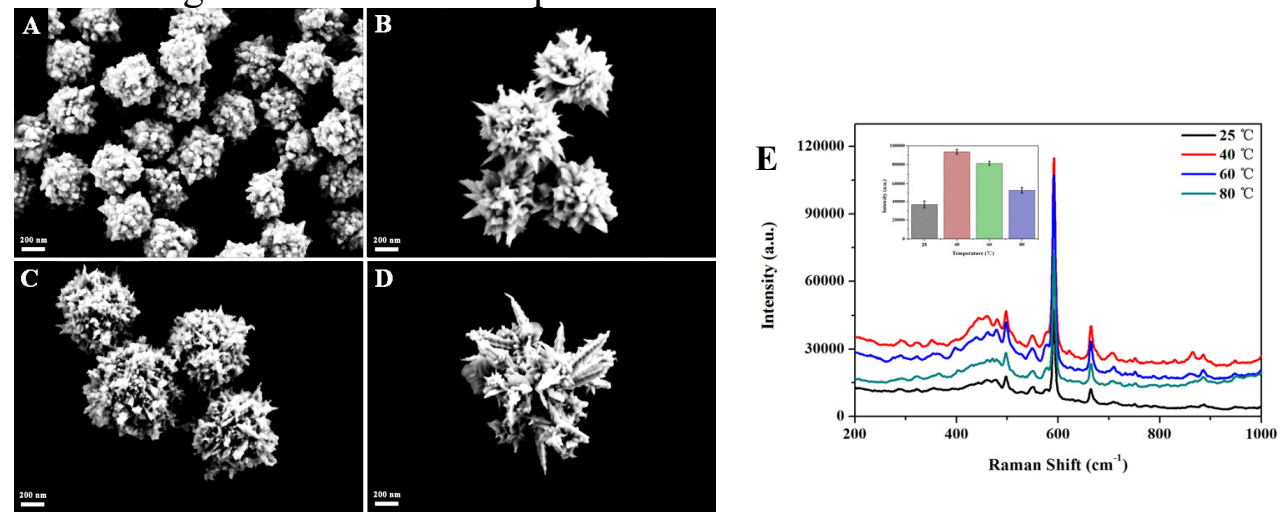

Fig. 4. The SEM images of the representatives of HU-GNPs under different bath temperature. (A) $25^{\circ} \mathrm{C}$, (B) $40^{\circ} \mathrm{C}$,

(C) $60{ }^{\circ} \mathrm{C}$, (D) $80^{\circ} \mathrm{C}$. (E) The SERS spectra of hollow urchin-like gold nanoparticles under different bath temperature $\left(10^{-6} \mathrm{M}\right.$ NBA$)$. The illustration was the SERS signals strength at $592 \mathrm{~cm}^{-1}$.

\subsubsection{HU-GNPs under optimal reaction conditions}

Through the experimental optimization, $100 \mu \mathrm{L} \mathrm{Ag} \mathrm{seeds,} 500 \mu \mathrm{L} \mathrm{HAuCl} 4$ were added under $40{ }^{\circ} \mathrm{C}$. And the HU-GNPs were characterized by SEM and SERS. As shown in Figure 5, the prepared nanoparticles possessed hollow urchins, and have good SERS enhanced effect. Besides, HU-GNPs were placed after different days to detect their SERS signals, and the results showed that they possessed good stability.
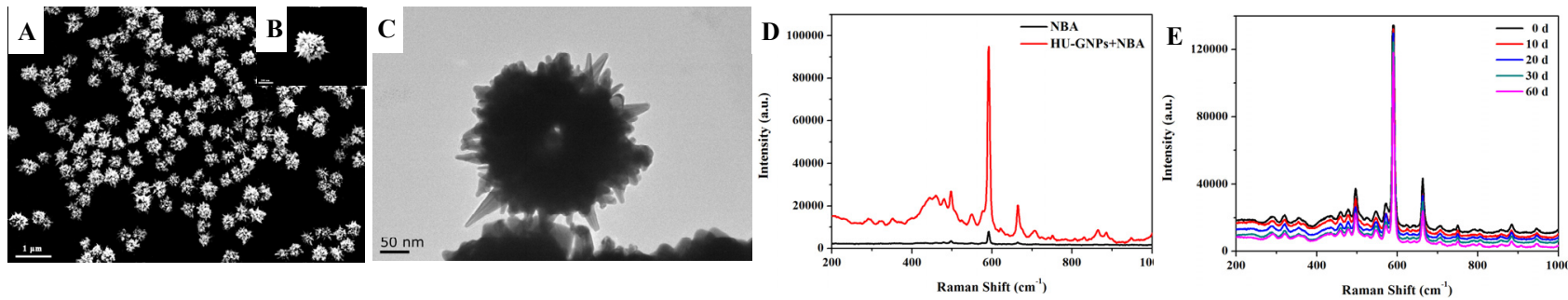

Fig. 5. (A-C) The SEM and TEM images of the representatives of HU-GNPs. (D) The SERS spectrum of HU-GNPs.

(E) The SERS spectra of HU-GNPs substrate placed after different time $(0 \mathrm{~d}, 5 \mathrm{~d}, 10 \mathrm{~d}, 20 \mathrm{~d}$ and $30 \mathrm{~d})$. The SERS spectra were recorded under experimental condition (785 nm excitation wavelength, $10 \mathrm{~s}$ integration time). 


\subsubsection{The intensity of SERS signals}

The SERS substrates were prepared with HU-GNPs via electrostatic self-assembly method under the assist of amine-terminated ITO glasses. The SERS spectra of different concentrations of NBA was collected. As can be seen in Figure 6, with the increasing of NBA concentrations (from $10^{-9} \mathrm{M}$ to $10^{-5}$ $\mathrm{M})$, the SERS intensity increased.

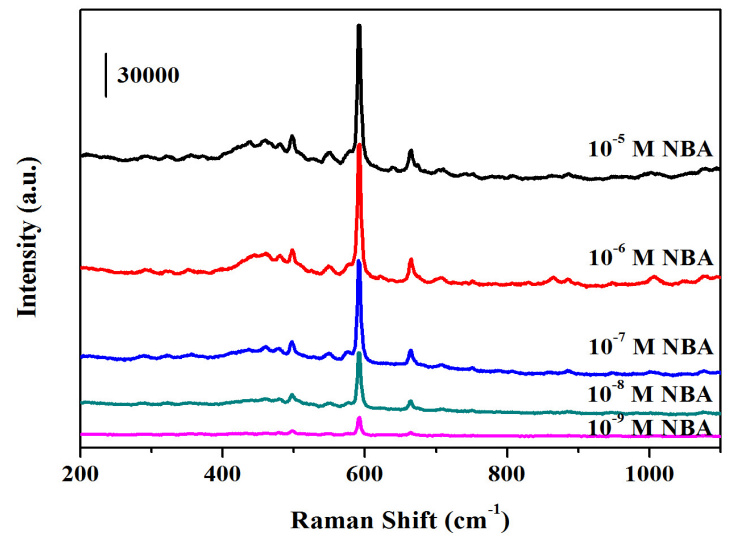

Fig. 6. (A) The SERS spectra of NBA under different concentrations from $10^{-9} \mathrm{M}$ to $10^{-5} \mathrm{M}$.

\section{Summary}

Using seed-mediated growth method, HU-GNPs with good morphology, uniform size, and excellent SERS enhancement were synthesized successfully. And by electrostatic self-assembly, the SERS substrates were prepared, and they exhibited high SERS activity, which means HU-GNPs substrates can be further applied in biomedical and bioscience fields.

\section{Acknowledgement}

This research was financially supported by the National Natural Science Foundation of China (Grant 21175022) and the Ministry of Science \& Technology of China (Grant 2012AA022703 and 2015AA020502).

\section{References}

[1] G. Maiorano, Monodispersed and size-controlled multibranched gold nanoparticles with nanoscale tuning of surface morphology. Nanoscale, vol. 3, pp. 2227-2232.A, 2011.

[2] Y. Sun, Shape-controlled synthesis of gold and silver nanoparticles. Science, vol. 298, 2002.

[3] B. Sharma, Bisboronic acids for selective, physiologically relevant direct glucose sensing with surface-enhanced Raman spectroscopy. J. Am. Chem. Soc, vol. 138(42), pp: 13952-13959, 2016.

[4] E. N. Esenturk, Surface-enhanced Raman scattering spectroscopy via gold nanostar. J. Raman Spectrosc, vol. 40, pp: 86-91, 2009.

[5] M. Käll, Plasmonic nanosensors: Inverse sensitivity. Nature Materials, vol.11,pp:570-571, 2012.

[6] L. Alfonso, Glutathione-triggered drug release from nanostructures. Current Topics in Medicinal Chemistry, vol. 14, pp: 2662-2671, 2014.

[7] W. Wang, Facile synthesis of hollow urchin-like gold nanoparticles and their catalytic activity[J]. Gold Bulletin, vol. 45(2), pp: 91-98, 2012.

[8] F. Xu, Facile synthesis of urchin-like gold submicrostructures for nonenzymatic glucose sensing[J]. Talanta, vol. 82(5), pp: 1845-1852, 2010. 